Portland State University

PDXScholar

\title{
A Biological Comparison of Two Sites: an Ecological Approach Using Community Ecology Metrics Based on Insect Faunal Composition
}

Kennedy J. Martin

Portland State University

Follow this and additional works at: https://pdxscholar.library.pdx.edu/honorstheses

Let us know how access to this document benefits you.

\section{Recommended Citation}

Martin, Kennedy J., "A Biological Comparison of Two Sites: an Ecological Approach Using Community Ecology Metrics Based on Insect Faunal Composition" (2017). University Honors Theses. Paper 419. https://doi.org/10.15760/honors.415

This Thesis is brought to you for free and open access. It has been accepted for inclusion in University Honors Theses by an authorized administrator of PDXScholar. Please contact us if we can make this document more accessible: pdxscholar@pdx.edu. 


\begin{abstract}
A biological comparison of two sites:
An ecological approach using community ecology metrics

based on insect faunal composition
\end{abstract}

by

Kennedy J. Martin

An undergraduate honors thesis submitted in partial fulfillment of the

requirements for the degree of

Bachelor of Science

in

University Honors

and

Organismal Biology

Thesis Adviser

Luis A. Ruedas

Portland State University

2017 


\title{
A biological comparison of two sites: An ecological approach using community ecology metrics based on insect faunal composition
}

\author{
ABSTRACT \\ Two natural sites in the Portland urban area managed by the City of Portland's Parks and \\ Recreation Department, River View Natural Area (RVNA) and Powers Marine Park (PMP), \\ were sampled to assess their insect faunal composition with the intention of determining whether \\ the areas are sufficiently similar ecologically that they could potentially be managed as a single \\ unit. The two areas were compared using community ecology metrics based on their respective \\ invertebrate fauna. The results suggested that RVNA and PMP had statistically different \\ ecological communities. The Shannon Diversity Index for PMP was 0.303, and 0.819 for \\ RVNA. The Horn Index of Community Overlap suggested a 93.46\% overlap based on these \\ data; however, a Hutcheson's t-test for community ecology data suggested significant \\ differences ( $\mathrm{P}<<<0.0001)$ between PMP and RVNA's insect faunal compositions.
}

\section{INTRODUCTION}

Natural areas are vital for the conservation of biodiversity, particularly when these areas are located in more urbanized settings. When natural areas decline we lose components of nature that are essential to maintaining agriculture and ecosystems, as well those that potentially benefit human health (Dizney and Ruedas, 2009; Schwägerl, 2016). As our cities continue to expand and push further into nature, it is important to protect, if not increase, the land that is set aside for parks, forests, and other natural areas. The question of how to best care for such areas reflects a debate that is central to the work of many ecologists and conservation biologists.

The manner in which protected areas are managed is of concern to local taxpayers as it can be much more cost-effective to manage adjacent natural areas as a single unit. The effectiveness of protection strategies is often assessed by analyzing loss of natural cover, social-ecological spillover, and population diversity of both plant and animal species (Ament \& Cumming, 2016). High resolution ecological data are extremely useful in assessing the social-ecological dynamics surrounding natural areas.

For example, biomonitoring uses organisms in natural communities to gauge the impact by humans (or other) impacts on particular ecosystems. This information can then be used to develop and implement management plans for the studied areas. Insects are often used as bioindicators in these studies due to their sensitivity to environmental change. However, another advantage to using insects in biomonitoring includes their abundance and, in many instances, large population sizes (McGeogh, 1998). In addition, using plants or vertebrates for biomonitoring incurs a greater regulatory burden.

The present study, the analysis of insect population density and diversity, examination of plant species diversity, and estimated canopy cover, in a series of randomized plots, make up the 
bulk of the data for a potential management assessment of two natural or semi-natural areas owned by the City of Portland: Powers Marine Park and River View Natural Area.

Powers Marine Park and River View Natural Area are two adjacent areas split in two by a single busy road. Powers Marine Park lies between the Willamette River and SW Macadam Ave (Oregon State Road 43). River View Natural Area is located on the west side of SW Macadam Ave. This road also serves as an impediment to any potential flow of organisms between sites. RVNA and PMP are also connected by culverts meant to act as wildlife corridors. However, it unclear whether there is any flow of species or movement of species through these culverts between the two sites. The assessment presented herein was undertaken in order to assess the ecological community similarity between the two sites using community ecology data.

\section{MATERIALS AND METHODS}

\section{Site Description and Selection}

River View Natural Area encompasses 146 acres, with 7 streams running through the property. It is bordered by Lewis and Clark College to the south, residential neighborhoods to the west, River View cemetery to the north, and SW Macadam Ave (OR 43) to the east. Powers Marine Park is a long and narrow property of approximately 14.25 acres wedged between OR 43 and the Willamette River. The riparian floodplain is home to hardwoods and riparian adapted herbaceous plants. Just west of this floodplain lies a mixed coniferous-hardwood forest abutting OR 43. All plots from which samples were collected were within the riparian zone or at the interface between floodplain and forest, and encompassing both habitat types.

A map of each park was divided into grids $14.14 \mathrm{~m}$ by $7.07 \mathrm{~m}$ (Brower et al. 1998) and a random number generator used to select three plots in each park. Each plot was sketched and photographed from all corners. Location of each plant within a plot was documented on the main sketch of the area. All flora contained in the plot were identified and recorded (Table 2 and Table 4).

\section{Trees}

In each plot, trees over $10 \mathrm{~cm}$ in diameter at breast height were identified and documented on the main sketch of the area (Tables 1 and 3). The radius of the canopy for each tree was measured by estimating the average extent of the canopy from the ground and measuring the distance from the tree to that point (Table 1 and Table 3). Tree height was calculated using distance from the base and the angle to the top of the tree using a clinometer and measuring tape. 


\section{Invertebrate Sampling}

All invertebrates were captured using plastic pitfall traps. Each pitfall trap $(710 \mathrm{~mL}$ capacity, 10 $\mathrm{cm}$ diameter, $15 \mathrm{~cm}$ deep) was filled with a small amount of ethanol to euthanize and preserve captured invertebrates, as well as being covered by an elevated plastic lid in order to prevent flooding by rainwater. Traps were arranged in a web configuration (Parmenter et al. 2003), with 5 central traps and 12 radial spokes of 11 trap stations per spoke at $1 \mathrm{~m}$ intervals, for a total of 137 traps. After a trap array was set, it was resampled and reset every 48 hours for a total of 3 sample collections per plot.

\section{Sample Processing}

Samples were collected three times from each web array at RVNA and PMP. Each row of an array was collected into a single labeled cup. Samples were then rinsed with ethanol and inspected under magnification. Invertebrates were identified to the lowest possible taxonomic category, sorted into one of 16 taxonomic categories (Table 5), and placed into labelled, ethanol filled storage vials. The vials were stored until the final counts could be performed.

\section{Data Analysis}

The collected invertebrate samples from both RVNA and PMP were used to calculate the indices of species diversity using the Shannon Diversity Index, H' (Shannon, 1948). The Shannon Diversity Index is an information theoretic index; that is, it takes into consideration both the presence-absence of species as well as the abundance. A Hutcheson's t-test was applied in order to calculate the significance of similarity between the diversity indices of RVNA and PMP (Hutcheson, 1970). The Horn Index of Community Overlap was used to determine the proportional overlap between the two communities (Horn, 1966).

\section{RESULTS}

Plot Characteristics/Structural Analysis—River View Natural Area

\begin{tabular}{|l|l|l|l|l|l|}
\hline \multicolumn{2}{|c|}{ Plot 1. Geographic coordinates: $\mathbf{4 5 . 4 5 6 3 0 1}^{\circ} \mathbf{N}, \mathbf{1 2 2 . 6 7 3 8 2}^{\circ}$ W; Elevation: $\mathbf{1 4 0 m}$ (datum: WGS84) } \\
\hline Plant \# & Species (common name) & Diameter $(\mathrm{m})$ & Canopy Radius $(\mathrm{m})$ & Canopy Area $\left(\mathrm{m}^{2}\right)$ & Tree Height $(\mathrm{m})$ \\
\hline 1 & Big leaf maple & 0.4386 & 8.5 & 227.0 & 32.6 \\
\hline 2 & Pacific red cedar & 0.1705 & 2.4 & 18.1 & 10.9 \\
\hline 3 & Pacific red cedar & 0.2879 & 0.6 & 1.1 & 14.9 \\
\hline 4 & Big leaf maple & 0.3835 & 6.24 & 122.3 & 24.6 \\
\hline
\end{tabular}


Table 1, continued

\begin{tabular}{|c|c|c|c|c|c|}
\hline 5 & Big leaf maple & 0.5822 & 4 & \begin{tabular}{|l|}
50.3 \\
\end{tabular} & 28.9 \\
\hline 6 & $\begin{array}{l}\text { Big leaf maple } \\
\text { (snag) }\end{array}$ & 0.3566 & 0 & 0 & 19.4 \\
\hline \multicolumn{6}{|c|}{ Plot 2. Geographic coordinates: $45.456471^{\circ} \mathrm{N}, \mathbf{1 2 2 . 6 7 1 7 9 2}^{\circ} \mathrm{W}$, Altitude: $122 \mathrm{~m}$ (datum: WGS84) } \\
\hline Plant \# & Species & Diameter (m) & $\begin{array}{l}\text { Canopy Radius } \\
\text { (m) }\end{array}$ & Canopy Area (m) & Tree Height $(\mathrm{m})$ \\
\hline 1 & Big leaf maple & 53.6 & 10.3 & 333.3 & 39.7 \\
\hline 2 & Big leaf maple & 69.9 & 6.7 & 141.0 & 52.9 \\
\hline 3 & Western hemlock & 11.9 & 1.9 & 11.3 & 11.0 \\
\hline \multicolumn{6}{|c|}{ 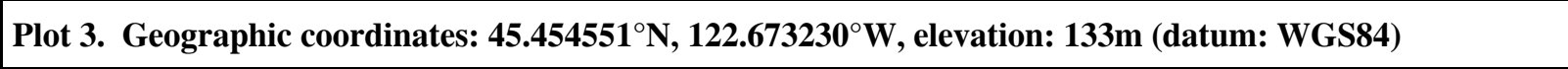 } \\
\hline Plant \# & Species & Diameter (m) & $\begin{array}{l}\text { Canopy Radius } \\
\text { (m) }\end{array}$ & Canopy Area (m) & Tree Height (m) \\
\hline 1 & Big leaf maple & 24.8 & 8.53 & 228.6 & 28.9 \\
\hline 2 & Douglas fir & 14.9 & 4.35 & 59.4 & 12.5 \\
\hline 3 & Western hemlock & 89 & 5 & 78.5 & 42.0 \\
\hline 4 & Pacific red cedar & 25.1 & 4.1 & 52.8 & 6.5 \\
\hline
\end{tabular}

Table 2: Plant species found in RVNA plots.

\begin{tabular}{|l|l|}
\hline \multicolumn{1}{|c|}{ Scientific Name } & \multicolumn{1}{c|}{ Common Name } \\
\hline Acer circinatum & Vine Maple \\
\hline Acer macrophyllum & Big Leaf Maple \\
\hline Athyrium sp. & Lady Fern \\
\hline Gaultheria shallon & Salal \\
\hline Mahonia aquifolium & Oregon Grape \\
\hline Maianthemum racemosum & False Solomon's Seal \\
\hline Polypodium glycyrrhiza & Licorice Fern \\
\hline Polystichum munitum & Sword Fern \\
\hline Pseudotsuga menziessii & Douglas Fir \\
\hline Rhamnus purshiana & Cascara Buckthorn \\
\hline Rosa gymnocarpa or R. nutkana & Baldhip Rose or Nookta Rose \\
\hline Symphoricarpos albus & Snowberry \\
\hline Thuja plicata & $\begin{array}{l}\text { Western Red Cedar } \\
\text { (Oregon Cedar, Canoe cedar) }\end{array}$ \\
\hline
\end{tabular}


Table 2, continued.

\begin{tabular}{|l|l|}
\hline Tusga heterophylla & Western Hemlock or (Pacific Hemlock) \\
\hline Vaccinium parvifolium Sm. & Red Huckleberry \\
\hline Unknown plants (4 species) & \\
\hline
\end{tabular}

\section{Plot Characteristics/Structural Analysis-Powers Marine Park}

Table 3: Plot location and trees in PMP.

Plot 1. Geographic coordinates: $45.45678^{\circ} \mathrm{N}, \mathbf{1 2 2 . 6 6 2 8}^{\circ} \mathrm{W}$, elevation: $21 \mathrm{~m}$ (datum: WGS 84)

\begin{tabular}{|l|l|l|l|l|l|}
\hline Plant \# & Species & Diameter $(\mathrm{m})$ & Canopy Radius $(\mathrm{m})$ & Canopy Area $(\mathrm{m})$ & Tree Height $(\mathrm{m})$ \\
\hline 1 & Pacific willow & $\begin{array}{l}0.325 \\
(\text { at } 4.65 \mathrm{~m} \text { from base })\end{array}$ & 8.5 & 227.65 & 12.28 \\
\hline 2 & Pacific willow & $\begin{array}{l}0.48 \\
\text { (at 4.84m from base }\end{array}$ & 2.4 & 18.09 & 9.00 \\
\hline
\end{tabular}

Plot 2. Geographic coordinates: WGS84: $45.45819^{\circ} \mathrm{N}, \mathbf{1 2 2 . 6 6 4 0 3}^{\circ} \mathrm{W}$, elevation 21 m (datum: WGS 84)

\begin{tabular}{|l|l|l|l|l|l|}
\hline Plant \# & Species & Diameter $(\mathrm{m})$ & Canopy Radius $(\mathrm{m})$ & Canopy Area $(\mathrm{m})$ & Tree Height $(\mathrm{m})$ \\
\hline 1 & Pacific willow & 0.267 & 8 & 200.96 & 9.25 \\
\hline 2 & Pacific willow & 0.332 & 7.1 & 158.29 & 15.64 \\
\hline 3 & Pacific willow & 0.18 & 3.5 & 38.465 & 6.50 \\
\hline
\end{tabular}

Plot 3. Geographic coordinates: $45.454551^{\circ} \mathrm{N}, \mathbf{1 2 2 . 6 7 3 2 3 0}^{\circ} \mathrm{W}$, elevation $21 \mathrm{~m}$ (datum: WGS84)

\begin{tabular}{|l|l|l|l|l|l|}
\hline Plant \# & Species & Diameter $(\mathrm{m})$ & Canopy Radius $(\mathrm{m})$ & Canopy Area $(\mathrm{m})$ & Tree Height $(\mathrm{m})$ \\
\hline 1 & Oregon ash & 0.15 & 5.4 & 91.56 & 11.20 \\
\hline 2 & White alder & 0.339 & 9.3 & 271.58 & 26.64 \\
\hline 3 & White alder & 0.315 & 8.3 & 216.31 & 36.26 \\
\hline 4 & White alder & 0.314 & 10.4 & 339.62 & 17.92 \\
\hline 5 & $\begin{array}{l}\text { Black } \\
\text { cottonwood }\end{array}$ & 0.424 & 9.06 & 257.73 & 39.10 \\
\hline 6 & $\begin{array}{l}\text { Black } \\
\text { cottonwood }\end{array}$ & 0.175 & $0(\mathrm{snag})$ & 0 & 14.64 \\
\hline 7 & $\begin{array}{l}\text { Black } \\
\text { cottonwood }\end{array}$ & 0.212 & 6.25 & 122.65 & 14.64 \\
\hline
\end{tabular}


Table 4: Plant species found in PMP plots.

\begin{tabular}{|l|l|}
\hline \multicolumn{1}{|c|}{ Scientific Name } & \multicolumn{1}{c|}{ Common Name } \\
\hline Alnus rhombifolia & White Alder \\
\hline Athyrium Roth & Lady Fern \\
\hline Cornus sericea & Red Twig Dogwood \\
\hline Fraxinus latifolia & Oregon Ash \\
\hline Geum macrophyllum & Large-leaved Geum \\
\hline Hedera helix & English Ivy \\
\hline Impatiens capensis & Spotted Jewel Weed \\
\hline Mentha pulegium & Pennyroyal \\
\hline Populus trichocarpa & Black Cottonwood \\
\hline Quercus garryana & Oregon White Oak (not verified) \\
\hline Rhamnus purshiana & Cascara Buckthorn \\
\hline Rubus ursinus & Pacific Blackberry \\
\hline Salix lucida & Pacific Willow \\
\hline Solanum dulcamara & Bittersweet Nightshade \\
\hline Symphyotrichum Subspicatum & Douglas' Aster \\
\hline Trametes versicolor & Turkey Tails \\
\hline Urtica dioica & Stinging Nettle \\
\hline Viburnum ellipticum & Oregon Viburnum \\
\hline Unknown A & Sedge (not verified) \\
\hline Unknown B & Cone Flower-Type Plant (not verified) \\
\hline Unknown C & Bush-like with Brown pods (not verified) \\
\hline Unknown D & Waxy Leafed Ground Cover (not verified) \\
\hline
\end{tabular}




\section{Faunal Characterics}

A total of 9835 invertebrates were collected in the two sites (Table 5). Of these, arthropods were identified to order, with the exception of Coleoptera and Arachnida, which were identified to family. However, some Coleoptera had to be placed in the "other" category due to lack of taxonomic expertise. Non-arthropod invertebrates also were classified as "other," for the same reason.

In the RVNA site we collected 2957 invertebrates, while in the PMP site we collected 7010. The only group that was specific to one site only was the arachnid Order Pseudoscorpiones, which were found only at the RVNA site. All other orders and families were found at both sites, although in differing densities (Tables 6-7; Fig. 1).

Table 5: Invertebrates collected (total numbers of each taxon) at RVNA and PMP.

\begin{tabular}{|c|c|c|c|c|}
\hline & & RVNA & PMP & TOTAL \\
\hline \multirow{3}{*}{ Coleoptera } & Carabidae & 635 & 707 & 1342 \\
\hline & Curculionidae & 2 & 2 & 4 \\
\hline & Other & 22 & 97 & 119 \\
\hline Diptera & & 66 & 53 & 119 \\
\hline Hymenoptera & & 10 & 15 & 25 \\
\hline Acari & & 252 & 30 & 282 \\
\hline Araneae & & 321 & 111 & 432 \\
\hline Pseudoscorpiones & & 9 & 0 & 9 \\
\hline Opiliones & & 3 & 16 & 19 \\
\hline Orthoptera & & 2 & 8 & 10 \\
\hline Collembola & & 921 & 5853 & 6774 \\
\hline Isopoda & & 520 & 23 & 543 \\
\hline $\begin{array}{l}\text { Hemiptera/ } \\
\text { Hemoptera }\end{array}$ & & 24 & 6 & 30 \\
\hline Myriapoda & & 55 & 19 & 74 \\
\hline
\end{tabular}


Table 5, continued.

\begin{tabular}{|c|c|c|c|c|}
\hline $\begin{array}{c}\text { Unidentified } \\
\text { Larvae }\end{array}$ & 24 & 29 & 53 \\
\hline Other & 91 & 41 & 132 \\
\hline TOTAL & & $\mathbf{2 9 5 7}$ & $\mathbf{7 0 1 0}$ & $\mathbf{9 8 3 5}$ \\
\hline Shannon Index & $\mathbf{0 . 8 1 8 5}$ & $\mathbf{0 . 3 0 3 1}$ & $\boldsymbol{P}<<<\mathbf{0 . 0 0 0 1}$ \\
\hline Horn index & & & & $\mathbf{0 . 9 3 4 6}$ \\
\hline
\end{tabular}

Table 6: Estimates of density at center of research plots, in individuals per square meter, calculated using the program "Distance" v. 7.0 (Thomas et al. 2010), for each of the taxa under consideration in either site.

\begin{tabular}{|c|c|c|c|c|c|}
\hline & & $\begin{array}{c}\text { Density } \\
\text { estimate }\end{array}$ & Standard Error & Lower $95 \%$ CI & Upper $95 \%$ CI \\
\hline \multirow{2}{*}{ Carabidae } & RVNA & 152.39 & 43.10 & 86.58 & 268.24 \\
\hline & PMP & 169.67 & 46.74 & 97.73 & 294.58 \\
\hline \multirow{2}{*}{ Curculionidae } & RVNA & 2.88 & 0.68 & 1.78 & 4.67 \\
\hline & PMP & 2.88 & 0.68 & 1.78 & 4.67 \\
\hline \multirow{2}{*}{ Other Coleoptera } & RVNA & 7.04 & 2.96 & 2.99 & 16.58 \\
\hline & PMP & 27.93 & 10.32 & 13.28 & 58.75 \\
\hline \multirow{2}{*}{ Diptera } & RVNA & 21.12 & 6.06 & 11.88 & 37.56 \\
\hline & PMP & 13.88 & 4.88 & 6.87 & 28.02 \\
\hline \multirow{2}{*}{ Hymenoptera } & RVNA & 4.11 & 1.29 & 2.17 & 7.79 \\
\hline & PMP & 7.20 & 2.34 & 3.68 & 14.07 \\
\hline \multirow{2}{*}{ Acari } & RVNA & 72.57 & 22.82 & 38.67 & 136.21 \\
\hline & PMP & 7.85 & 2.85 & 3.80 & 16.23 \\
\hline \multirow{2}{*}{ Araneae } & RVNA & 77.04 & 21.72 & 43.83 & 135.39 \\
\hline & PMP & 26.64 & 9.47 & 13.11 & 54.12 \\
\hline \multirow{2}{*}{ Pseudoscorpiones } & RVNA & 5.184 & 1.35 & 3.058 & 8.79 \\
\hline & PMP & 0.00 & 0.00 & 0.00 & 0.00 \\
\hline \multirow{2}{*}{ Opiliones } & RVNA & 4.32 & 1.76 & 0.93 & 20.12 \\
\hline & PMP & 11.52 & 3.39 & 6.30 & 21.06 \\
\hline \multirow{2}{*}{ Orthoptera } & RVNA & 5.76 & 1.36 & 3.55 & 9.34 \\
\hline & PMP & 4.61 & 2.04 & 1.72 & 12.35 \\
\hline \multirow{2}{*}{ Collembola } & RVNA & 221.00 & 99.25 & 90.25 & 541.29 \\
\hline & PMP & 1404.60 & 550.97 & 642.25 & 3072.00 \\
\hline \multirow{2}{*}{ Isopoda } & RVNA & 124.79 & 40.05 & 65.84 & 236.54 \\
\hline & PMP & 8.28 & 2.74 & 4.24 & 16.16 \\
\hline \multirow{2}{*}{$\begin{array}{l}\text { Hemiptera and } \\
\text { Homoptera }\end{array}$} & RVNA & 7.68 & 2.68 & 3.80 & 15.54 \\
\hline & PMP & 3.46 & 1.00 & 1.92 & 6.23 \\
\hline
\end{tabular}


Table 6, continued.

\begin{tabular}{|l|l|r|r|r|r|}
\hline \multirow{2}{*}{ Myriapoda } & RVNA & 13.20 & 3.92 & 7.30 & 23.87 \\
\cline { 2 - 6 } & PMP & 9.12 & 4.57 & 3.07 & 27.11 \\
\hline \multirow{2}{*}{$\begin{array}{l}\text { Unidentified } \\
\text { larvae }\end{array}$} & RVNA & 6.91 & 2.99 & 2.88 & 16.58 \\
\cline { 2 - 6 } & PMP & 10.44 & 3.97 & 4.79 & 22.75 \\
\hline \multirow{2}{*}{ Other invertebrates } & RVNA & 26.21 & 9.77 & 12.37 & 55.51 \\
\cline { 2 - 6 } & PMP & 14.76 & 6.04 & 6.36 & 34.25 \\
\hline
\end{tabular}

Table 7: Estimates of total number of individuals present in the research plots (area $=124.14$ $\mathrm{m}^{2}$ ), in individuals, calculated using the program "Distance" v. 7.0 (Thomas et al. 2010), for each of the taxa under consideration in either site.

\begin{tabular}{|c|c|c|c|c|c|}
\hline & & $\begin{array}{c}\text { Population } \\
\text { estimate }\end{array}$ & Standard Error & Lower $95 \%$ CI & Upper $95 \%$ CI \\
\hline \multirow{2}{*}{ Carabidae } & RVNA & 18,918 & $5,350.90$ & 10,747 & 33,300 \\
\hline & PMP & 21,063 & $5,802.50$ & 1,232 & 36,569 \\
\hline \multirow{2}{*}{ Curculionidae } & RVNA & 358 & 84.31 & 220 & 580 \\
\hline & PMP & 358 & 84.31 & 220 & 580 \\
\hline \multirow{2}{*}{$\begin{array}{l}\text { Other } \\
\text { Coleoptera }\end{array}$} & RVNA & 874 & 367.54 & 371 & 2,058 \\
\hline & PMP & 3,468 & $1,281.20$ & 1,649 & 7,293 \\
\hline \multirow{2}{*}{ Diptera } & RVNA & 2,622 & 752.27 & 1,474 & 4,663 \\
\hline & PMP & 1,723 & 605.39 & 853 & 3,478 \\
\hline \multirow{2}{*}{ Hymenoptera } & RVNA & 511 & 160.61 & 270 & 967 \\
\hline & PMP & 894 & 291.23 & 457 & 1,747 \\
\hline \multirow{2}{*}{ Acari } & RVNA & 9,009 & $2,832.30$ & 4,800 & 16,909 \\
\hline & PMP & 975 & 353.50 & 472 & 2,014 \\
\hline \multirow{2}{*}{ Araneae } & RVNA & 9,563 & $2,696.90$ & 5,441 & 16,807 \\
\hline & PMP & 3,307 & $1,176.10$ & 1,628 & 6,719 \\
\hline \multirow{2}{*}{$\begin{array}{l}\text { Pseudoscorpio } \\
\text { nes }\end{array}$} & RVNA & 644 & 167.69 & 380 & 1,091 \\
\hline & PMP & 0 & 0.00 & 0 & 0 \\
\hline \multirow{2}{*}{ Opiliones } & RVNA & 536 & 218.76 & 115 & 2,496 \\
\hline & PMP & 1,430 & 421.08 & 782 & 2,614 \\
\hline \multirow{2}{*}{ Orthoptera } & RVNA & 715 & 168.38 & 441 & 1,160 \\
\hline & PMP & 572 & 253.29 & 213 & 1,533 \\
\hline \multirow{2}{*}{ Collembola } & RVNA & 27,439 & $12,321.00$ & 11,204 & 67,197 \\
\hline & PMP & 174,370 & $68,398.00$ & 79,730 & 381,360 \\
\hline \multirow{2}{*}{ Isopoda } & RVNA & 15,492 & $4,972.00$ & 8,173 & 29,364 \\
\hline & PMP & 1,028 & 339.70 & 526 & 2,007 \\
\hline \multirow{2}{*}{$\begin{array}{l}\text { Hemiptera and } \\
\text { Homoptera }\end{array}$} & RVNA & 953 & 332.68 & 471 & 1,929 \\
\hline & PMP & 429 & 123.77 & 238 & 773 \\
\hline \multirow{2}{*}{ Myriapoda } & RVNA & 1,639 & 486.88 & 906 & 2,964 \\
\hline & PMP & 1,132 & 567.16 & 381 & 3,365 \\
\hline \multirow{2}{*}{$\begin{array}{l}\text { Unidentified } \\
\text { larvae }\end{array}$} & RVNA & 858 & 371.43 & 358 & 2,058 \\
\hline & PMP & 1,296 & 493.46 & 595 & 2,824 \\
\hline
\end{tabular}


Table 7, continued.

\begin{tabular}{|l|l|r|r|r|r|}
\hline \multirow{2}{*}{$\begin{array}{l}\text { Other } \\
\text { invertebrates }\end{array}$} & RVNA & 3,253 & $1,213.00$ & 1,536 & 6,891 \\
\cline { 2 - 6 } & PMP & 1,832 & 749.89 & 790 & 4,251 \\
\hline
\end{tabular}

Figure 1: Relative abundance curves depicting number of individuals per species, showing species diversity and dominance. Horizontal axis: Species, ordered in sequence from high to low number of individuals; Vertical axis: number of individuals in species. Graph B graphically demonstrates the higher species diversity at RVNA, while graph A (PMP) shows a more typical, but also highly diverse, relative abundance curve with, however, higher dominance of a few species.

\section{A: PMP}

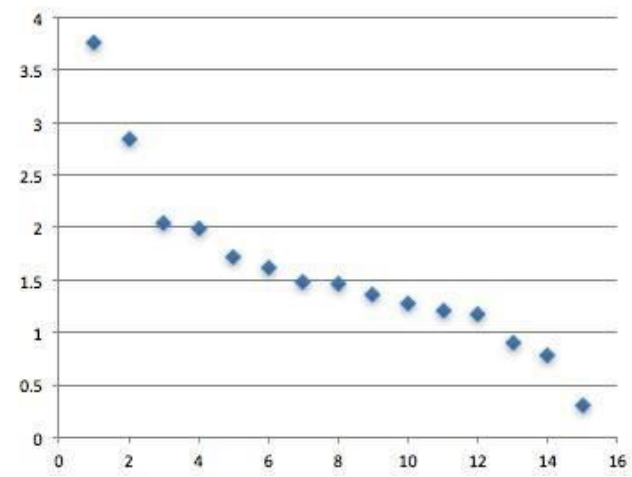

B: RVNA

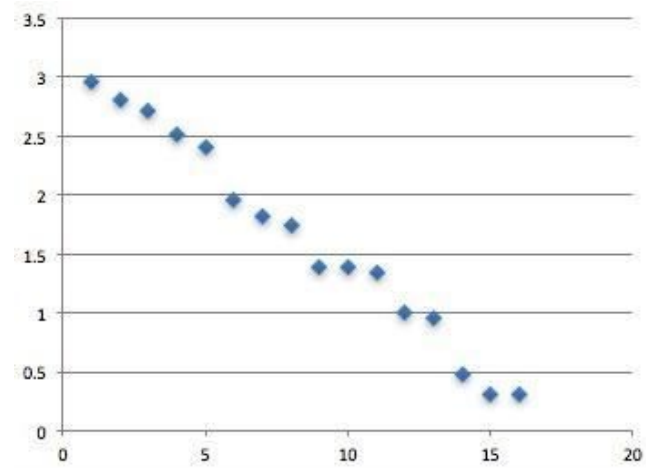

\section{Statistical Analyses}

The Shannon Diversity Index for Powers Marine Park was 0.303, and 0.819 for River View Natural Area (Table 5). Hutcheson's t-test for community data was 51.95, corresponding to $P$ $<<0.0001$. This indicates a highly significant difference between the two sites based on community composition of insect species.

\section{DISCUSSION}

Differences in the insect faunal composition between sites is presumably influenced by differences in flora and substrate. Tree and plant species varied between PMP and RVNA. The PMP site is located directly next to the Willamette River in a riparian zone, and is dominated primarily by Salix lucida. Assorted shrubs are also present, as well as an understory cover of herbs. The RVNA site contains coniferous and deciduous forest filled with an abundance of Acer macrophyllum. Both RVNA and PMP contain 4 species of trees larger than $10 \mathrm{~cm}$ in diameter. 
However, these species are mutually exclusive (Table 1 and Table 3 ). Nineteen trees and plants species (combined) were found in RVNA and 22 were recorded in PMP, only two of which were the same species (Table 2 and Table 4). The greater diversity of invertebrates and also flora inhabiting the PMP site suggests the nutrient richness of the soil also may be different (Table 5).

A statistical analysis indicates a significant difference in community ecology metrics in species diversity between the two sites of interest. The Shannon Diversity index quantifies diversity by combining number of species present in a community with abundance and summarizing them with a numerical value between zero and one. The value found at the PMP site, $\mathrm{H}^{\prime}=0.304$, indicates relatively lower diversity in comparison to the RVNA site, $\mathrm{H}^{\prime}=0.819$. However, it is probable that the H' value found at the PMP site was strongly affected by a numerical dominance of Collembola, which numerically overpowered all the other taxa. The $\mathrm{P}$ value derived from the Hutcheson's t-test underscores the statistically significant difference between sites. The relative abundance curve (Figure 1) graphically displays a fairly even level of species dominance at RVNA, while PMP maintains a level of high diversity but displays a curve representative of a higher dominance by a fewer select species. The Horn index of community overlap gave a calculated value of $93.46 \%$ which emphasized the similarity in insect species composition between the two sites.

Given that the data collected indicates significant differences in their respective insect faunal compositions, the two sites are not ecologically identical. As a result we can conclude that they should be managed as two separate units.

\section{LITERATURE CITED}

Ament, J. M., \& Cumming, G. S.. 2016 . Scale dependency in effectiveness, isolation, and social-ecological spillover of protected areas. Conservation Biology, 30(4), 846-855.

Brower, James E., Jerrold H. Zar, and Carl Von. Ende. 1998. Field and Laboratory Methods for General Ecology. Boston, MA: WCB McGraw-Hill.

Horn, H. S. 1966. Measurement of overlap in comparative ecological studies. American Naturalist, 100(914):419-423.

Hutcheson, K. 1970. A test for comparing diversities based on the Shannon formula. Journal of Theoretical Biology, 29:151-154.

Margalef, R. 1957. La teoría de la información en ecología. Memorias de la Real Academia de Ciencias y Artes de Barcelona, 32:373-449.

McGeogh, M. A.. 1998. The selection, testing and application of terrestrial insects as bioindicators. Biological Reviews, 73(2), 181-201.

Parmenter, R. R., T. L. Yates, D. R. Anderson, K. P. Burnham, J. L. Duunum, A. B. Franklin, M. T. Friggens, B. C. Lubow, M. Miller, G. S. Olson, C. A. Parmenter, J. Pollard, E. Rexstad, T. M. Shenk, T. R. Stanley, and G. C. White. 2003. Small-mammal density 
estimation: A field comparison of grid-based vs. web-based density estimators. Ecological Monographs, 73(1):1-26.

Shannon, C. E. 1948. A mathematical theory of communication. Bell System Technical Journal, 27:379-423.

Schwägerl, Christian. 2016. Vanishing Act: What's Causing Sharp Decline in Insects and Why It Matters: Yale Environment 360. (n.d.).

Simpson, E. H. 1949. Measurement of diversity. Nature, 163:688.

Thomas, L., S. T. Buckland, E. A. Rexstad, J. L. Laake, S. Strindberg, S. L. Hedley, J. R. B. Bishop, T. A. Marques, and K. P. Burnham. 2010. Distance software: design and analysis of distance sampling surveys for estimating population size. Journal of Applied Ecology 47:5-14.

Zar, J. H. 2010. Biostatistical Analysis, 5th ed. Upper Saddle River, New Jersey: Prentice Hall/Pearson, xiii+944 pp. 